Supporting Information

\title{
Living Polymer Networks Based on a RAFT Crosslinker: toward 3D and 4D Printing Applications
}

Ali Bagheri ${ }^{\mathrm{a}, \mathrm{b}^{*}}$, Honglei Ling ${ }^{\mathrm{b}}$ Chris William Anderson Bainbridge ${ }^{\mathrm{b}}$ and Jianyong Jin ${ }^{\mathrm{b} *}$

a School of Science and Technology, The University of New England, Armidale NSW 2351, Australia

b School of Chemical Sciences, The University of Auckland, Auckland 1010, New Zealand

Email: Ali.bagheri@une.edu.au

Email: j.jin@auckland.ac.nz 


\section{Experimental section}

\subsection{Materials}

2-Hydroxyethyl acrylate (HEA), 2-Hydroxyethyl methacrylate (HEMA), $n$-butyl acrylate (BA) and oligo (ethylene glycol) methyl ether acrylate (OEGA) (average $M_{n}=480 \mathrm{gmol}^{-1}$ ) were purchased from SigmaAldrich and they were filtered through basic alumina to remove inhibitors. Poly (ethylene glycol) diacrylate (PEGDA) (average $M_{n}=250$ gmol $^{-1}$ ), 5, 10, 15, 20-Tetraphenyl-21H, 23H-porphine zinc (ZnTPP) and $N$ isopropylacrylamide (NIPAAm) were purchased from Sigma-Aldrich and used as received. $O$-nitrobenzyl methacrylate (NBMA) monomer was purchased from Polyscinces and used as received. Thionyl chloride was purchased from Honeywell Riedel-de-Haën, and used as received. (2,2'(Carbonothioyldisulfanediyl)bis(2-methylpropanoic acid)) (TTC-diacid) was purchased from Apollo Scientific and used as received. All the other reagents were used as received unless otherwise specified.

\subsection{Characterization}

${ }^{1} \mathrm{H}-{ }^{13} \mathrm{C}$ heteronuclear single quantum coherence spectroscopy (HSQC) and nuclear magnetic resonance (NMR) spectra were recorded on the Bruker DXR400 (400 MHz) and Bruker DXR300 (300 MHz) spectrophotometer. Chemical shifts are reported in parts per million (ppm) referenced to $\delta 7.26 \mathrm{ppm}$ and $77.0 \mathrm{ppm}$ from chloroform or $\delta 2.50$ and $39.5 \mathrm{ppm}$ from DMSO for ${ }^{1} \mathrm{H}$ and ${ }^{13} \mathrm{C}$ respectively. The multiplicities of ${ }^{1} \mathrm{H}$ signals are designated by the following abbreviations: $\mathrm{s}=$ singlet; $\mathrm{d}=\mathrm{doublet}$; $\mathrm{dd}=$ doublet of doublets; $\mathrm{m}=$ multiplet; br = broad. All ${ }^{13} \mathrm{C}$ NMR spectra were acquired using broadband decoupled mode, and assignments were determined using DEPT sequences.

Total TTC-XL monomer conversions of the prepared networks were measured based on the mass of the dry samples (after extensive washing and drying in the room temperature).

Photopolymerization was performed with a pair of LumiLED 5W RGB E27 mount LED lamps positioned on opposite sides of the sample, parallel to the benchtop and each $10 \mathrm{~cm}$ from the sample. Below is the simple diagram of the photopolymerisation setup:

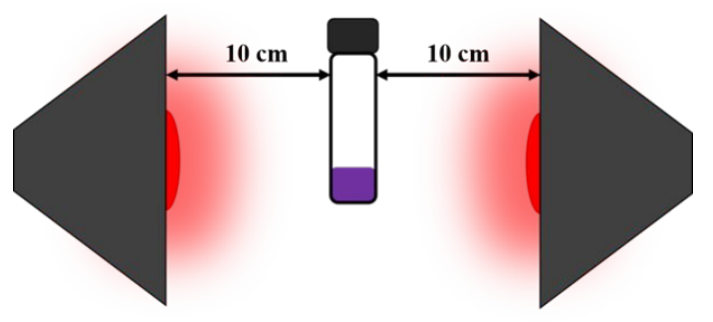


The swelling ratio of the networks were defined as $W_{w} / W_{d}$, where $W_{w}$ is the weight of a sample swollen in a specified solvent; $W_{d}$ is the weight of the sample in the dry state. The mass of the dry samples were determined after extensive washing in different solvents (such as DMSO, MeCN and tetrahydrofuran (THF)) for a minimum of $24 \mathrm{~h}$ and drying in the room temperature. Mass increase percentage of the modified networks were determined using the following equation:

$$
\text { Mass increase }(\%)=\frac{W_{d(\text { modified network })}-W_{d(\text { parent network })}}{W_{d(\text { parent network })}} \times 100
$$

\subsection{Procedure for Synthesis of TTC crosslinker (TTC-XL) using HEMA}

The TTC diacid $(1.0 \mathrm{~g}, 3.54 \mathrm{mmol})$ was added to thionyl chloride $(10.0 \mathrm{ml})$ at room temperature under a nitrogen atmosphere. The reaction was stirred at $50{ }^{\circ} \mathrm{C}$ for 5 hours. After cooling, the excessive thionyl chloride was removed under reduced pressure to give TTC acyl chloride as a yellow solid, which was then re-dissolved in DCM. The resulting solution was concentrated under reduced pressure again to obtain a yellow solid, which was used for the next step without further purification.

A solution of HEMA (1.15 g, $8.85 \mathrm{mmol})$ and pyridine $(700.04 \mathrm{mg}, 8.85 \mathrm{mmol})$ in anhydrous DCM (20.0 $\mathrm{ml}$ ) was prepared and the solution of TTC acyl chloride (1.13 g, $3.54 \mathrm{mmol})$ in anhydrous DCM (20.0 ml) was added dropwise at $0{ }^{\circ} \mathrm{C}$ under nitrogen atmosphere. The reaction was allowed to warm to room temperature and stirred overnight under a nitrogen atmosphere. Water $(40.0 \mathrm{ml})$ was poured into the reaction and the water phase was extracted by DCM three times. The organic phase was combined and washed with brine once, dried with anhydrous $\mathrm{Na}_{2} \mathrm{SO}_{4}$. The mixture was filtered and the filtrate was concentrated under reduced pressure to give the crude product, which was purified using chromatographic column, with eluent of hexane/ethyl acetate (v/v 8:1). The target compound (TTC-HEMA) was obtained as a yellow oil (792.37 mg, $1.56 \mathrm{mmol}, 44.2 \%) .{ }^{1} \mathrm{H}$ NMR (300 MHz, Chloroform-d) $\delta 6.17-6.05$ (m, 2H), $5.68-5.49(\mathrm{~m}, 2 \mathrm{H}), 4.34(\mathrm{~s}, 8 \mathrm{H}), 1.94(\mathrm{~s}, 6 \mathrm{H}), 1.65(\mathrm{~s}, 12 \mathrm{H}) .{ }^{13} \mathrm{C}$ NMR (100 MHz, Chloroform-d) $\delta 218.61$ , $172.58,167.13,136.04,126.20,63.63,62.21,56.20,25.20,18.40$.

\subsection{Procedure for Synthesis of TTC-XL using HEA}

The TTC diacid $(1.0 \mathrm{~g}, 3.54 \mathrm{mmol})$ was added to thionyl chloride $(10.0 \mathrm{ml})$ at room temperature under a nitrogen atmosphere. The reaction was stirred at $50{ }^{\circ} \mathrm{C}$ for 5 hours. After cooling, the excessive thionyl 
chloride was removed under reduced pressure to give TTC acyl chloride as a yellow solid, which was then re-dissolved in DCM. The resulting solution was concentrated under reduced pressure again to obtain a yellow solid, which was used for the next step without further purification.

A solution of HEA $(856.36 \mathrm{mg}, 7.08 \mathrm{mmol})$ and pyridine $(587.71 \mathrm{mg}, 7.43 \mathrm{mmol})$ in anhydrous DCM (5.0 $\mathrm{ml})$ was prepared and the solution of TTC acyl chloride $(565.11 \mathrm{mg}, 1.77 \mathrm{mmol})$ in anhydrous DCM (5.0 ml) was added dropwise at $0{ }^{\circ} \mathrm{C}$ under a nitrogen atmosphere. The reaction was allowed to warm to room temperature and stirred overnight under a nitrogen atmosphere. Water $(20.0 \mathrm{ml})$ was poured into the reaction and the water phase was extracted by DCM three times. The organic phase was combined and washed with brine once, dried with anhydrous $\mathrm{Na}_{2} \mathrm{SO}_{4}$. The mixture was filtered and the filtrate was concentrated under reduced pressure to give the crude product, which was purified using chromatographic column, with eluent of hexane/ethyl acetate (v/v 5:1). The target compound (TTC-HEA) was obtained as a yellow oil (419.32 mg, $0.88 \mathrm{mmol}, 49.5 \%$ ). ${ }^{1} \mathrm{H}$ NMR (400 MHz, Chloroform-d) $\delta 6.50-6.37$ (m, 2H), $6.20-6.06(\mathrm{~m}, 2 \mathrm{H}), 5.92-5.81(\mathrm{~m}, 2 \mathrm{H}), 4.38-4.30(\mathrm{~m}, 8 \mathrm{H}), 1.65-1.60(\mathrm{~m}, 12 \mathrm{H}) .{ }^{13} \mathrm{C}$ NMR $(100 \mathrm{MHz}$, Chloroform-d) $\delta 218.57,172.60,165.90,131.47,128.12$, $63.62,62.04,56.17,25.20$. HR-MS (ESI) calcd. 501.0691 for $\mathrm{C}_{19} \mathrm{H}_{26} \mathrm{NaO}_{8} \mathrm{~S}_{3}$, found $501.0682[\mathrm{M}+\mathrm{Na}]^{+}$.

\subsection{General Procedure for the Synthesis of PET-PNs; e.g.}

PN-1: In a typical experiment, the TTC-XL (1) (synthesized using HEMA) (55 mg, 0.108 mmol), ZnTPP (14.74 uL, $2.17 \times 10^{-5} \mathrm{mmol}$ of ZnTPP stock solution), and DMSO (217.4 uL) were charged to a glass vial and sealed with a rubber septum. The solution was bubbled with $\mathrm{N}_{2}$ for 30 minutes, and then irradiated under a red LED light $\left(635 \mathrm{~nm}, 0.7 \mathrm{~mW} / \mathrm{cm}^{2}\right)$ for 16 hours. The PN-1 was then washed in DMSO to remove ZnTPP and any unreacted monomers (monomer conversion of $\sim 72 \%$ ).

PN-2: PN-2 was obtained after synthesis of TTC-XL (2) using HEA and was then utilized as discrete pieces for the formation of a monolithic scaffold.

PN-3: In a typical experiment, the TTC-XL (synthesized using HEMA) (26 mg, 0.052 mmol), BA (65.9 mg, $0.52 \mathrm{mmol})$, ZnTPP (17.4 uL, $2.56 \times 10^{-5} \mathrm{mmol}$ from ZnTPP stock solution), and DMSO (114 uL) were charged to a glass vial and sealed with a rubber septum. The solution was bubbled with $\mathrm{N}_{2}$ for 30 minutes, and then irradiated under a red LED light for 48 hours (total monomer conversion $~ 72 \%$ ). The PN-3 was then washed in DMSO to remove ZnTPP and any unreacted monomers. 


\subsection{General Procedure for the Post-Synthesis Monomer Insertions into as Synthesized PET-PNs; e.g. Photo-Growth of PN-1:}

PN-1G-i: PN-1 was first soaked in a prepared solution containing; OEGA (1878 mg, $3.91 \mathrm{mmol})$, ZnTPP (132.6 uL, $1.95 \times 10^{-4}$ mmol from ZnTPP stock solution), and DMSO (780 uL) for $24 \mathrm{~h}$. The infiltrated PN-1 was then irradiated under a red LED light $\left(635 \mathrm{~nm}, 0.7 \mathrm{~mW} / \mathrm{cm}^{2}\right)$ for $2 \mathrm{~h}$ to afford PN-1G-i. The PN$1 \mathrm{G}-\mathrm{i}$ was then washed in DMSO to remove ZnTPP and any unreacted monomers. The OEGA monomer conversion was found to be $\sim 7.8 \%$.

PN-1G-ii: PN-1G-i was first cut into two pieces and one piece was soaked in a prepared solution containing; BA (485 mg, $3.78 \mathrm{mmol}$ ), ZnTPP (128.3 uL, $1.89 \times 10^{-4} \mathrm{mmol}$ from ZnTPP stock solution), and DMSO $(760 \mathrm{uL})$ for $24 \mathrm{~h}$. The infiltrated piece was then irradiated under a red LED light for $6 \mathrm{~h}$ to afford PN-1Gii. The PN-1G-ii was then washed in DMSO to remove ZnTPP and any unreacted monomers.

Note: The target degree of polymerization was calculated based upon simplified assumptions, e.g. the theoretical molecular weight $\left(M_{n}\right)$ of network strands were assumed comparable to that of linear polymer chains formed without crosslinking. Therefore, the target degree of polymerization for the post-synthesis monomer addition into the network-bound TTCs were calculated based on the theoretical $M_{n}$ :

$M_{n \text { network strands }}=\frac{[\text { Monomer }]}{[\mathrm{TTC}-\mathrm{XL}] \text { or }[\text { network }-T T C]} \times \mathrm{MW}$ monomer $\times \alpha+\mathrm{MW}$ TTC-XL

Where [Monomer], [TTC-XL] or [network-TTC], MW monomer, $\alpha$, and MW TTC correspond to initial moles of monomer (added for the post-synthesis transformation process), moles of TTC-XL or network-TTC, molecular mass of monomer, monomer conversion (based on the mass of the dry samples), and molecular mass of TTC-XL or network-TTC.

The moles of TTC in the PET-PNs used for the post-synthesis processes were calculated as follows:

$[\mathrm{TTC}]=($ Mass of PET-PN $) /\left(M_{n}\right.$ network strands $)$

Where Mass of PET-PN and $M_{n \text { network strands }}$ correspond to the mass of the PET-PN being used (mg), and the molecular mass of each strand in the network $(\mathrm{mg} / \mathrm{mmol})$. 


\section{Determination of degree of polymerization to fabricate $\mathrm{PN}-1 \mathrm{G}-\mathrm{i}$ :}

TTC-XL(1) monomer conversion in PN-1 $=72 \%$

$[$ TTC-XL $]=0.72 \times 0.108 \mathrm{mmol}=0.078 \mathrm{mmol}$

$[\mathrm{OEGA}] /[\mathrm{TTC}-\mathrm{XL}] /[\mathrm{ZnTPP}]=50: 1: 5 \times 10^{-3}$

$[\mathrm{OEGA}] /[\mathrm{TTC}-\mathrm{XL}] /[\mathrm{ZnTPP}]=[3.91] /[0.078] /\left[1.95 \times 10^{-4}\right]$

\section{Determination of degree of polymerization to fabricate PN-1G-ii:}

$M_{n \text { network strands }}=\frac{[\text { Monomer }]}{[\mathrm{TTC}]} \times \mathrm{MW}$ monomer $\times \alpha+\mathrm{MW}$ TTC-XL

$M_{n \text { network strands }}=\frac{[50]}{[1]} \times 480 \times 0.078+506$

$M_{n \text { network strands }}=2378 \mathrm{mg} / \mathrm{mmol}$

Weight of PN-1G-i $=179.9 \mathrm{mg}$

PN-1G-i was cut into two pieces, therefore the weight of each piece $\sim 90 \mathrm{mg}$

Moles of network-TTC $=(90 \mathrm{mg}) /(2378 \mathrm{mg} / \mathrm{mmol})=0.0378 \mathrm{mmol}$

$[\mathrm{BA}] /\left[\right.$ network-TTC]/[ZnTPP] $=100: 1: 5 \times 10^{-3}$

$[\mathrm{BA}] /\left[\right.$ network-TTC]/[ZnTPP] $=[3.78]:[0.0378]:\left[1.89 \times 10^{-4}\right]$

\section{Photo-Growth/Welding of PN-2:}

PN-2G/W: Small discrete pieces of PN-2 $(\sim 50 \mathrm{mg})$ were initially soaked in a prepared solution containing;

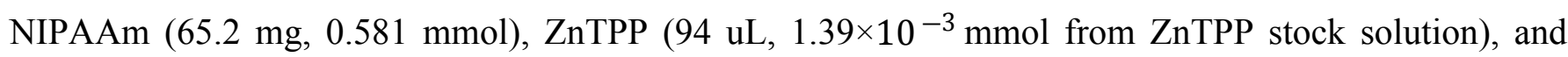
DMSO (700 uL) for $24 \mathrm{~h}$, while placed under a mild pressure $(\sim 2 \mathrm{kPa})$. These infiltrated pieces were then irradiated under a red LED light for $48 \mathrm{~h}$ to afford $\mathrm{PN}-2 \mathrm{G} / \mathrm{W}$. The modified network was repeatedly washed and swelled in DMSO, ethanol, acetonitrile and THF to remove ZnTPP and any unreacted monomers. 


\section{Photo-Growth/Functionalization of PN-3:}

PN-3G-PNBMA: PN-3 was first cut into two pieces and one piece soaked in a prepared solution containing; NBMA (127 mg, $0.58 \mathrm{mmol}), \mathrm{ZnTPP}\left(39.3 \mathrm{uL}, 5.8 \times 10^{-3} \mathrm{mmol}\right.$ from ZnTPP stock solution), and DMSO $(580 \mathrm{uL})$ for $24 \mathrm{~h}$. This piece was irradiated under a red LED light for $48 \mathrm{~h}$ to afford PN-3GPNBMA (NBMA monomer conversion $~ 25 \%$ ). The PN-3G-PNBMA was then washed in DMSO to remove ZnTPP and any unreacted monomers.

PN-3G- PNIPAAm: The other piece of PN-3 was soaked in a prepared solution containing; NIPAAm (65.4 mg, $0.58 \mathrm{mmol}$ ), ZnTPP (39.3 uL, 5.8 $\times 10^{-3} \mathrm{mmol}$ from ZnTPP stock solution), and DMSO (580 uL) for $24 \mathrm{~h}$. This piece was irradiated under a red LED light for $48 \mathrm{~h}$ to afford PN-3G- PNIPAAm (NIPAAm monomer conversion $\sim 18 \%$ ). The PN-3G- PNIPAAm was then washed in DMSO to remove ZnTPP and any unreacted monomers.

\section{Photo-Welding of NT-3-G/F-NBMA and PN-3G- PNIPAAm:}

PN-3W: The modified PN-3G-PNBMA and NT-3-G/F-NIPAM networks were soaked in a solution containing OEGA (3425 mg, $7.13 \mathrm{mmol})$, ZnTPP (241.9 uL, 3.5 $\times 10^{-4} \mathrm{mmol}$ from ZnTPP stock solution), and DMSO (700 uL) for $24 \mathrm{~h}$. These two pieces were put in contact (see figure S8) and irradiated under a red LED light for 48 hours to afford PN-3W. The welded network was repeatedly washed and swelled in DMSO, ethanol and acetonitrile to remove ZnTPP and any unreacted monomers.

\subsection{A layer by layer manufacturing of a PET-PN (3D-PN) using a modified DLP 3D printer equipped with a photomask and red LED light sources}

3D-PN: In a typical experiment, the TTC-XL(1) (synthesized using HEMA) (100 mg, $0.197 \mathrm{mmol}$ ), BA (1264 mg, $9.87 \mathrm{mmol}$ ), PEGDA (2467 mg, $9.87 \mathrm{mmol}$ ), ZnTPP (1351 uL, $1.99 \times 10^{-3} \mathrm{mmol}$ of ZnTPP stock solution), and DMSO (3987 uL) were charged to a glass vial and sealed with a rubber septum. The solution was bubbled with $\mathrm{N}_{2}$ for 30 minutes, and then transferred to a modified DLP 3D printerequiped with red LED lights and ma photomask under an inert $\mathrm{N}_{2}$ atmosphere. The 3D-PN was printed using the following predefined parameters: $20 \mu \mathrm{m}$ layers, $4 \mathrm{hr}$ exposure for the bottom 5 layers, and $2 \mathrm{hr}$ exposure for the remaining layers. The 3D-PN was then washed in DMSO to remove ZnTPP and any unreacted monomers. 


\subsection{General Procedure for the Post-Printing Transformation of PET-PNs; e.g. Photo-Growth of}

3D-PN:

3D-PN-G-PNIPAAm: 3D-PN was first soaked in a prepared solution containing; NIPAAm (311.3 mg, $2.75 \mathrm{mmol})$, ZnTPP (190.2 uL, 2.81 $\times 10^{-4} \mathrm{mmol}$ from ZnTPP stock solution), and DMSO (2806 uL) for $24 \mathrm{~h}$. The infiltrated network was then irradiated under a red LED light $\left(635 \mathrm{~nm}, 0.7 \mathrm{~mW} / \mathrm{cm}^{2}\right)$ for $2 \mathrm{~h}$ to afford NT-3D-G-PNIPAAm.

\section{Photo-Welding of NT-3D-G-PNIPAAm}

3D-PN-W: The modified NT-3D-G-PNIPAAm was first cut into two pieces and then the two halves of were soaked in a solution containing OEGA (3425 mg, $7.13 \mathrm{mmol})$, ZnTPP (241.9 uL, 3.5× $10^{-4} \mathrm{mmol}$ from ZnTPP stock solution),) and DMSO (700 uL) for $24 \mathrm{~h}$. These two pieces were put in contact and irradiated under a red LED light for 24 hours to afford 3D-PN-W. 

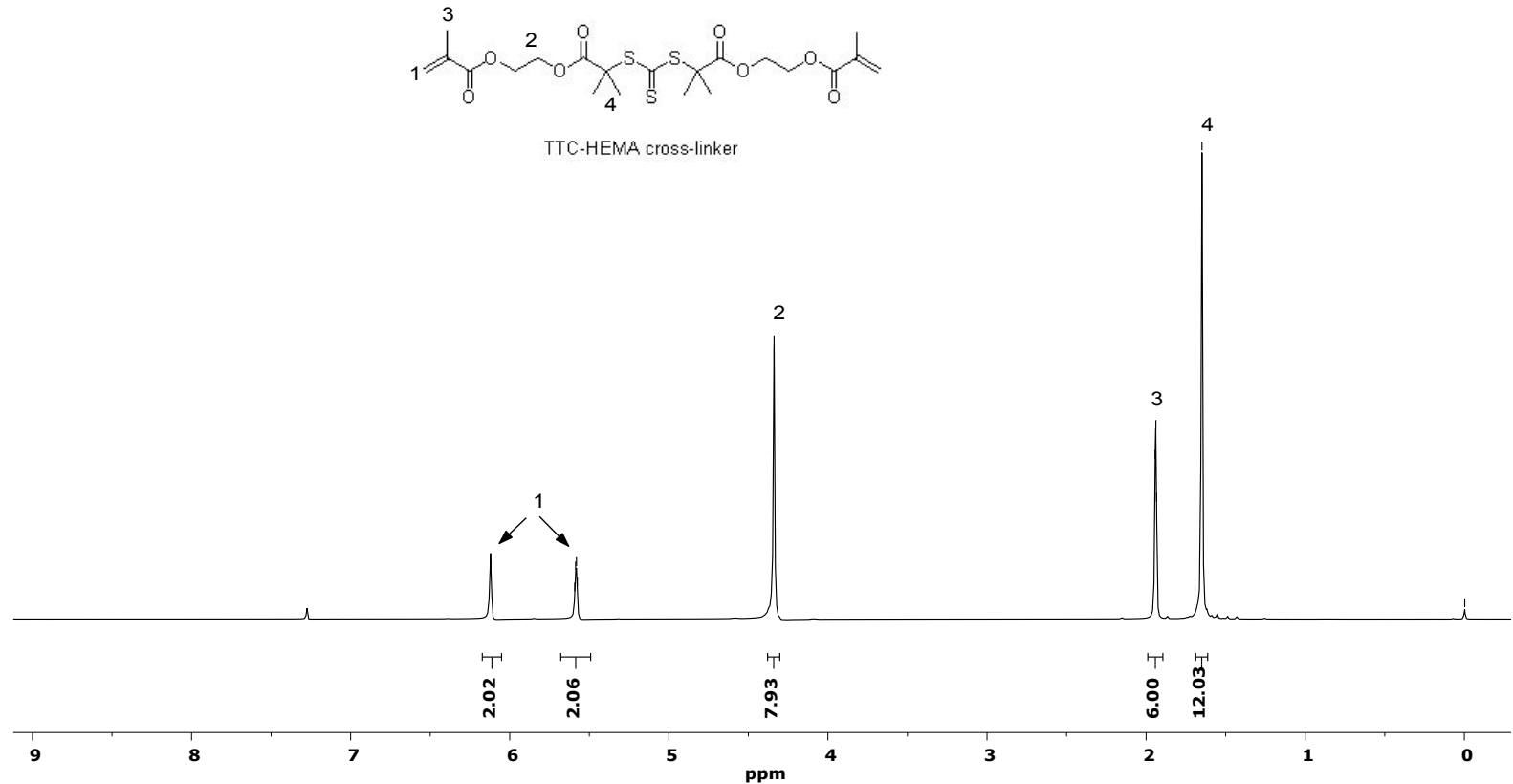

Figure S1. ${ }^{1} \mathrm{H}$ NMR of TTC-XL synthesized using HEMA 


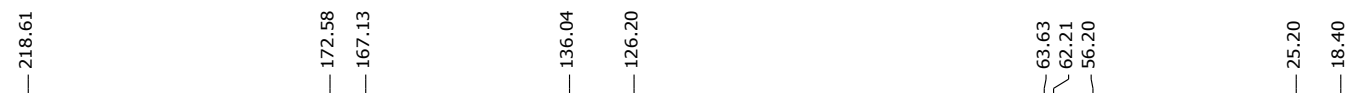

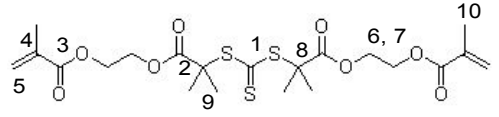

TTC-HEMA crosslinker
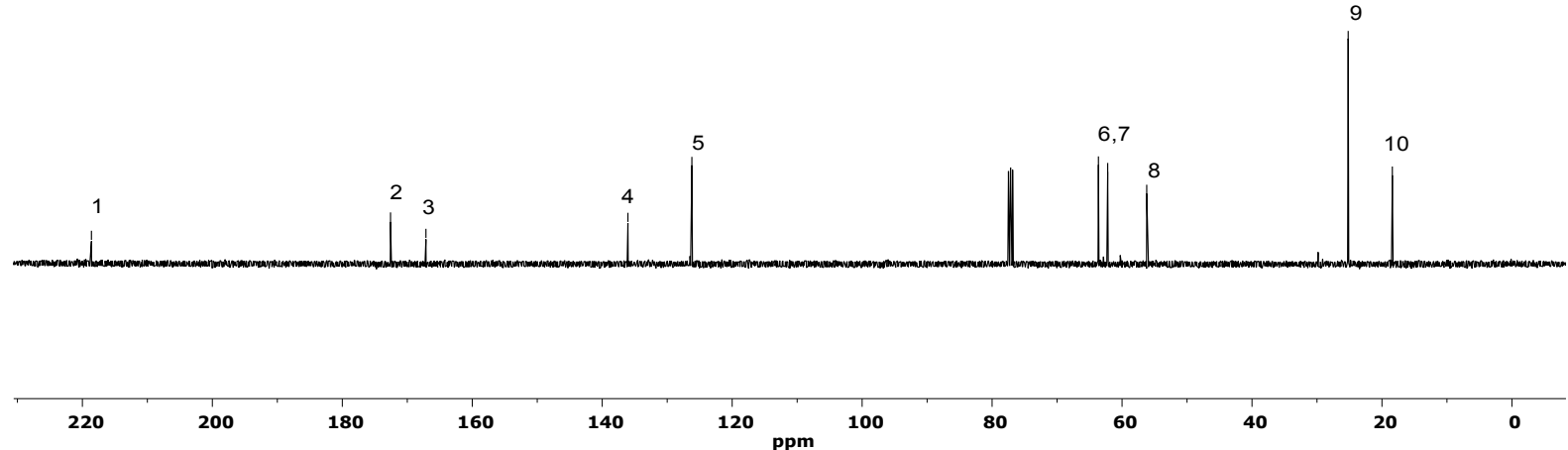

Figure S2. ${ }^{13} \mathrm{C}$ NMR of TTC-XL synthesized using HEMA. 


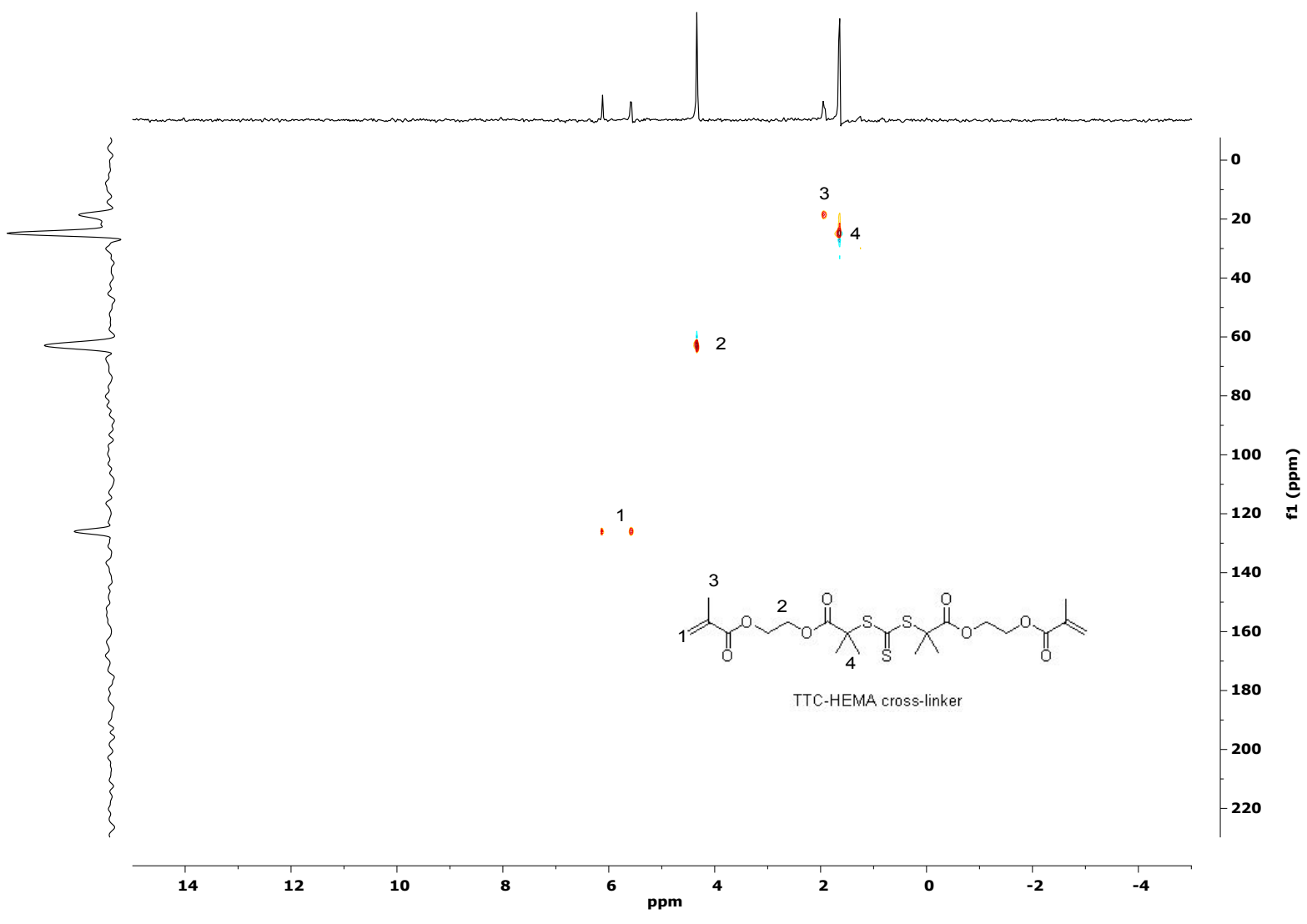

Figure S3. HSQC of TTC-XL synthesized using HEMA. 


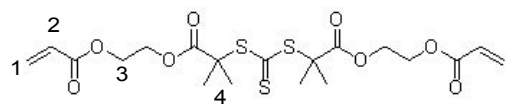

TTC-HEA cross-linker

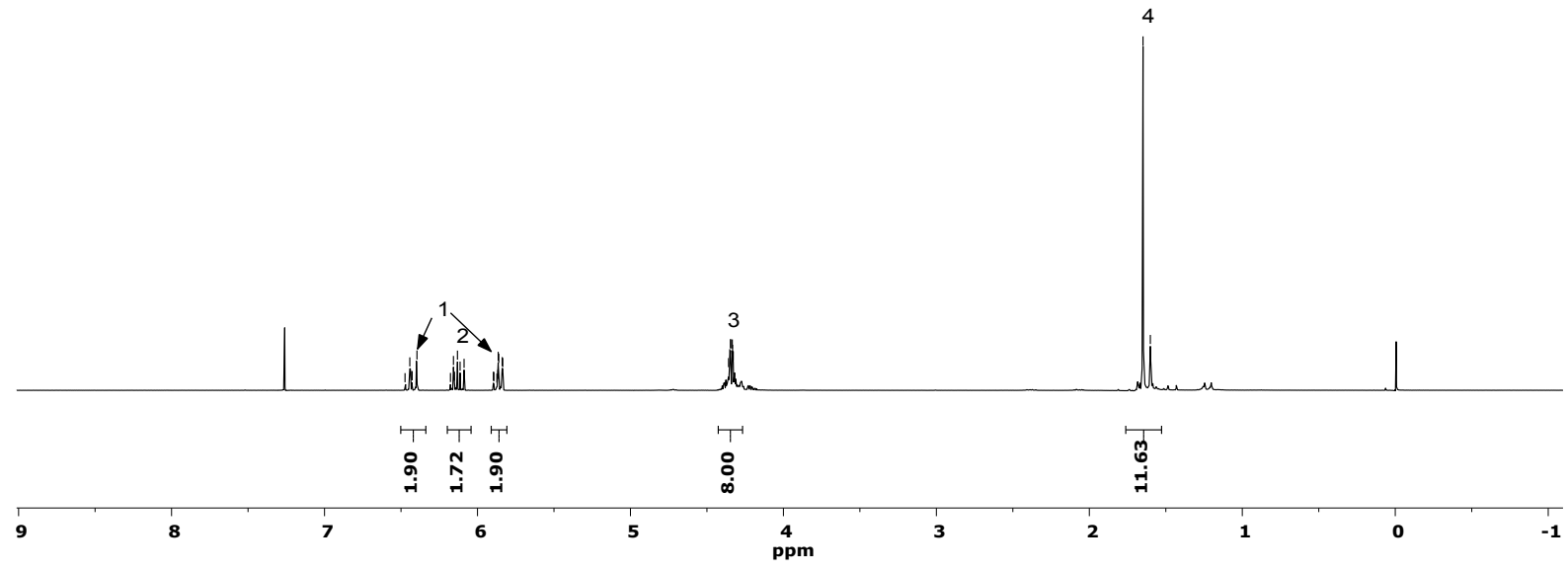

Figure S4. ${ }^{1} \mathrm{H}$ NMR of TTC-XL synthesized using HEA. 


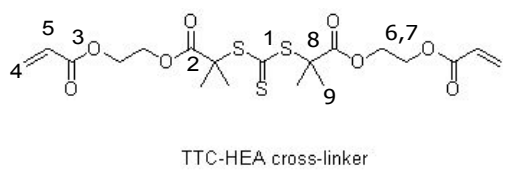

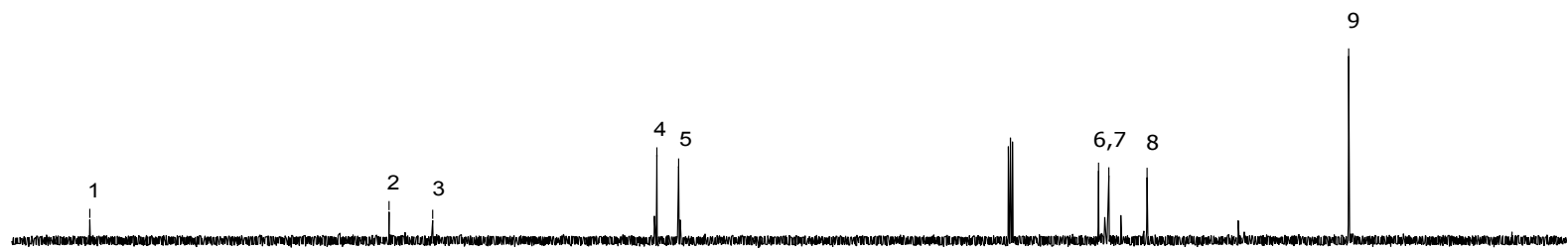

Figure S5. ${ }^{13} \mathrm{C}$ NMR of TTC-XL synthesized using HEA 


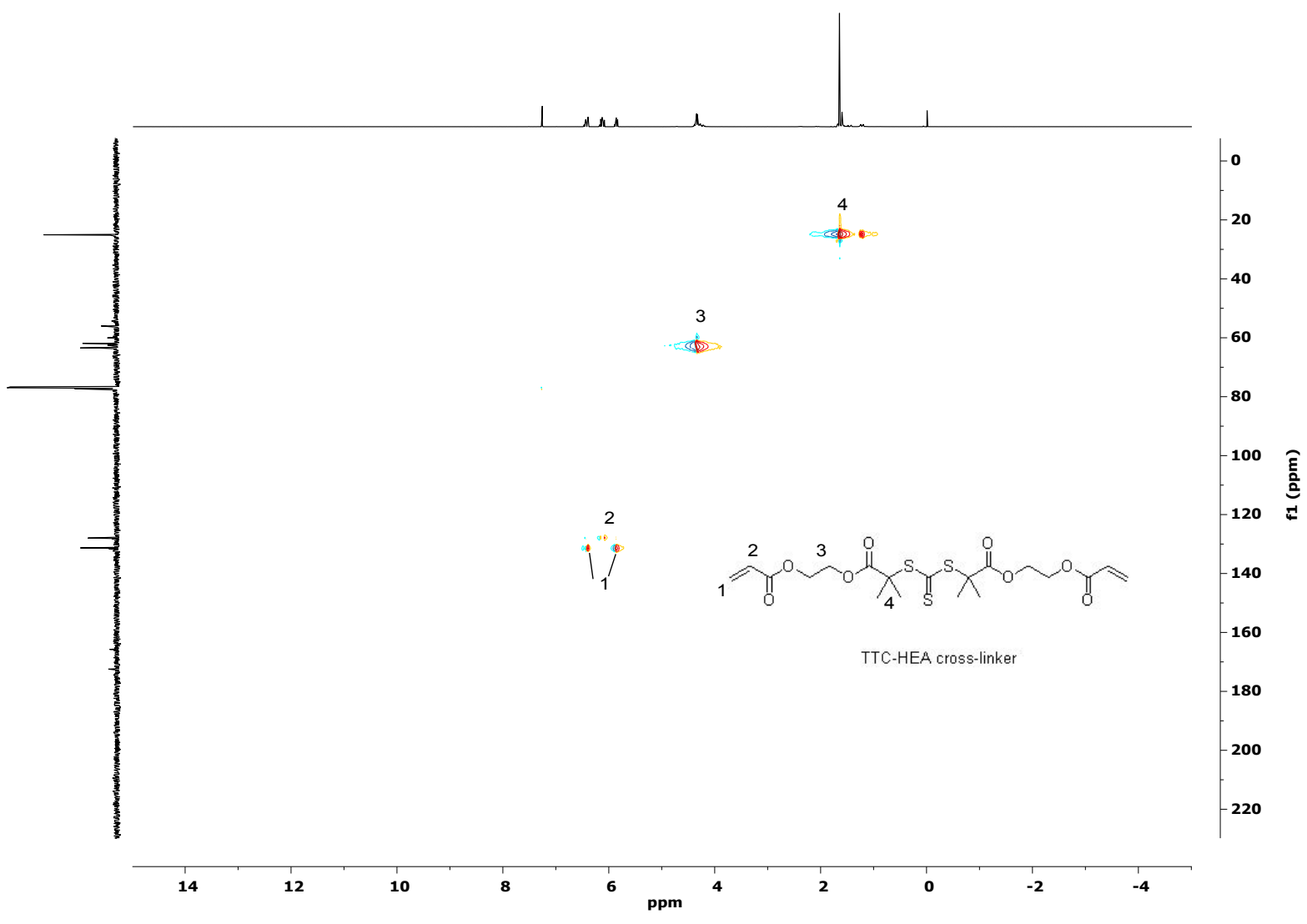

Figure S6. HSQC spectrum of TTC-XL synthesized with HEA. 


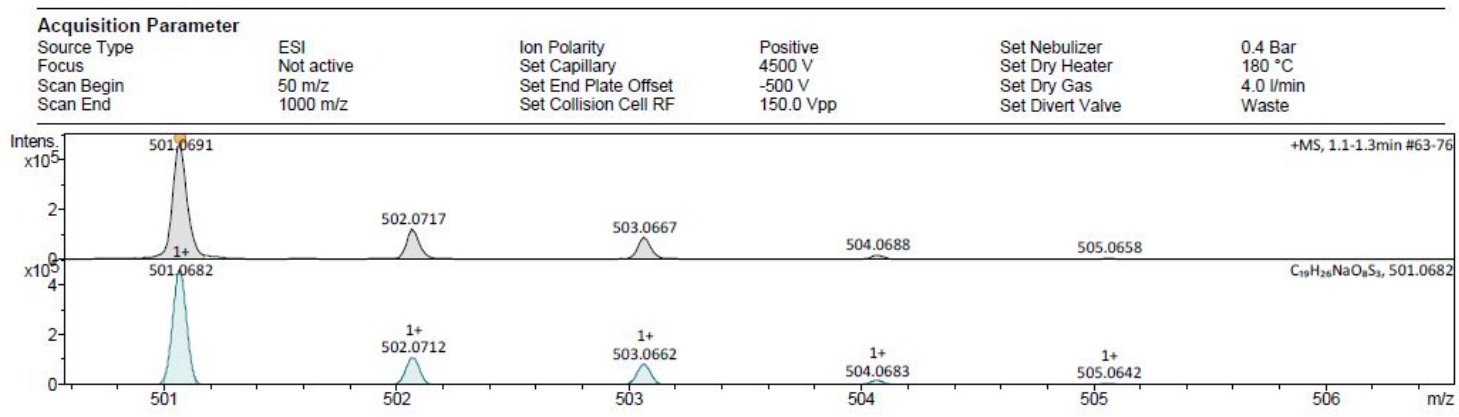

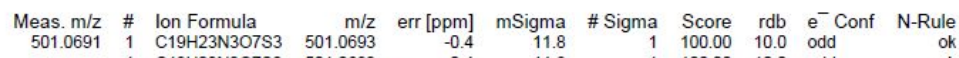
\begin{tabular}{llrrrrrrr}
$\mathrm{C} 19 \mathrm{H} 23 \mathrm{~N} 3 \mathrm{O} / \mathrm{S} 3$ & 501.0693 & -0.4 & 11.8 & 1 & 100.00 & 10.0 & odd & ok \\
\hline $\mathrm{C} 19 \mathrm{H} 26 \mathrm{NaO} 8 \mathrm{~S} 3$ & 501.0682 & 1.8 & 15.6 & 1 & 100.00 & 6.5 & even & ok
\end{tabular}

Figure S7. Mass spectrum of TTC-XL synthesized using HEA. 


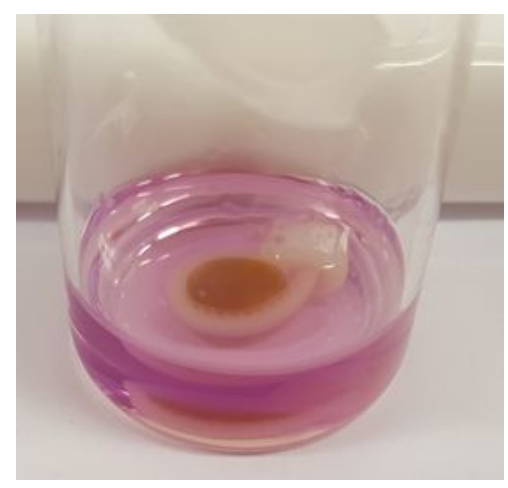

Figure S8. Optical image of "PN-3G-PNBMA" and "PN-3G- PNIPAAm" held together prior to the photowelding reaction. 


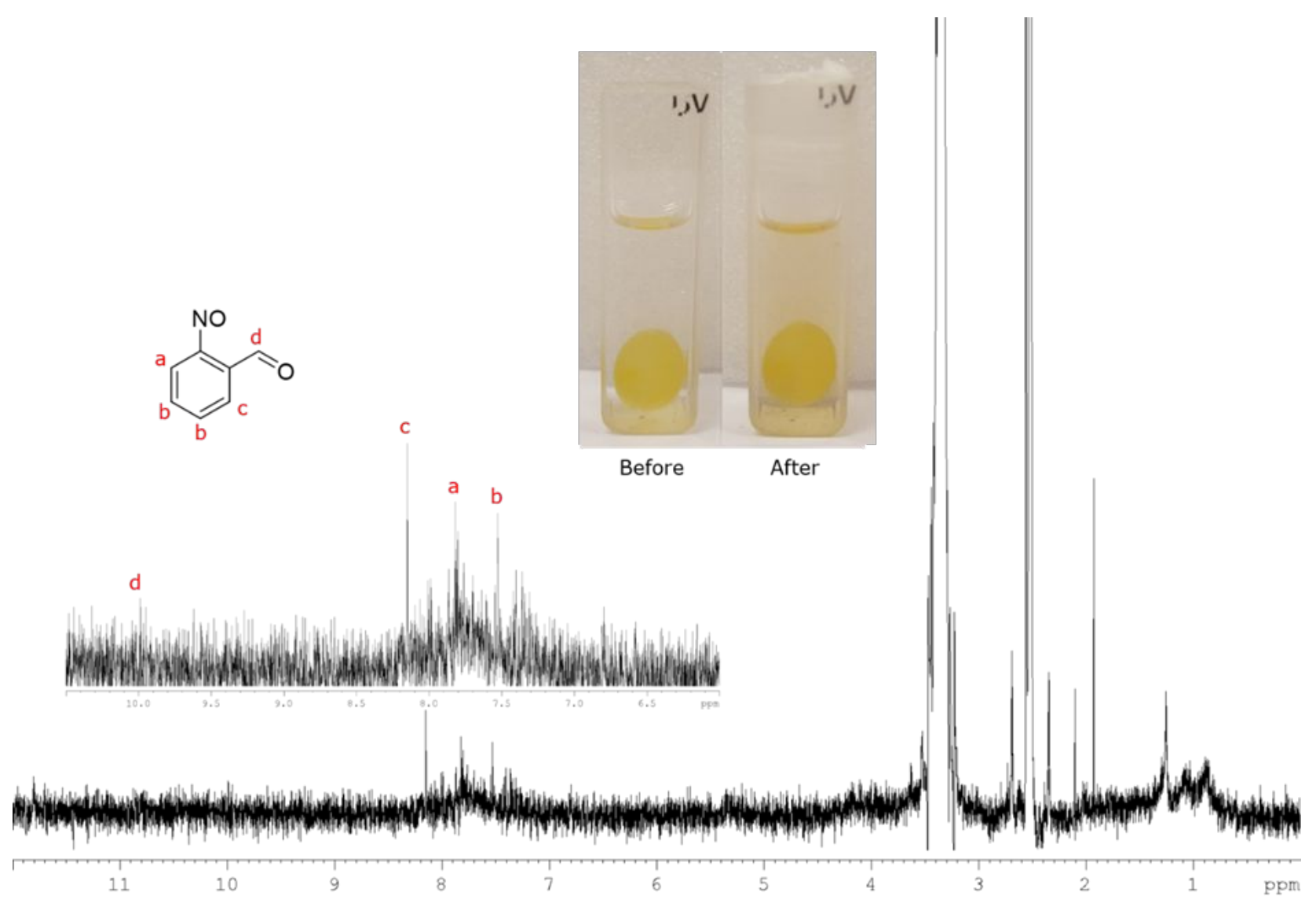

Figure S9. ${ }^{1} \mathrm{H}$ NMR of $o$-nitrobenzyl groups cleaved from the PN-3W under UV (356 nm) irradiation, with inset optical images of PN-3W before and after $o$-nitrobenzyl groups cleavage. 


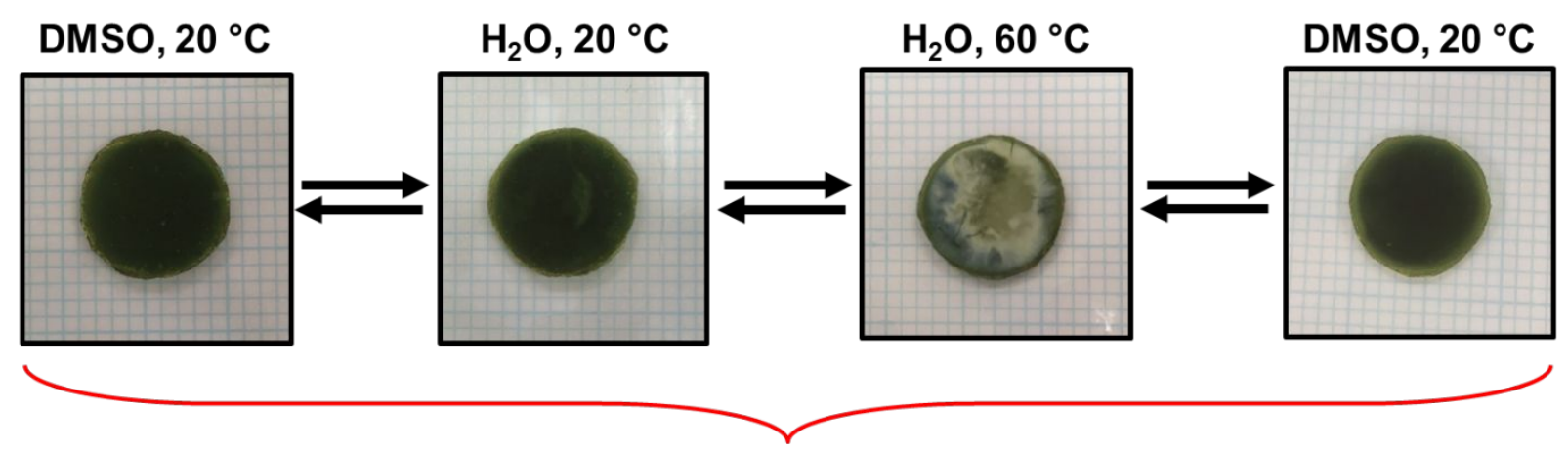

3D-PN-F-PNIPAAm

Figure S10. The optical images of the 3D-PN-F-PNIPAAm after solvent exchange and/or temperature variation. These optical images show the reversibility in opacity changes upon solvent exchange or water temperature variation. 

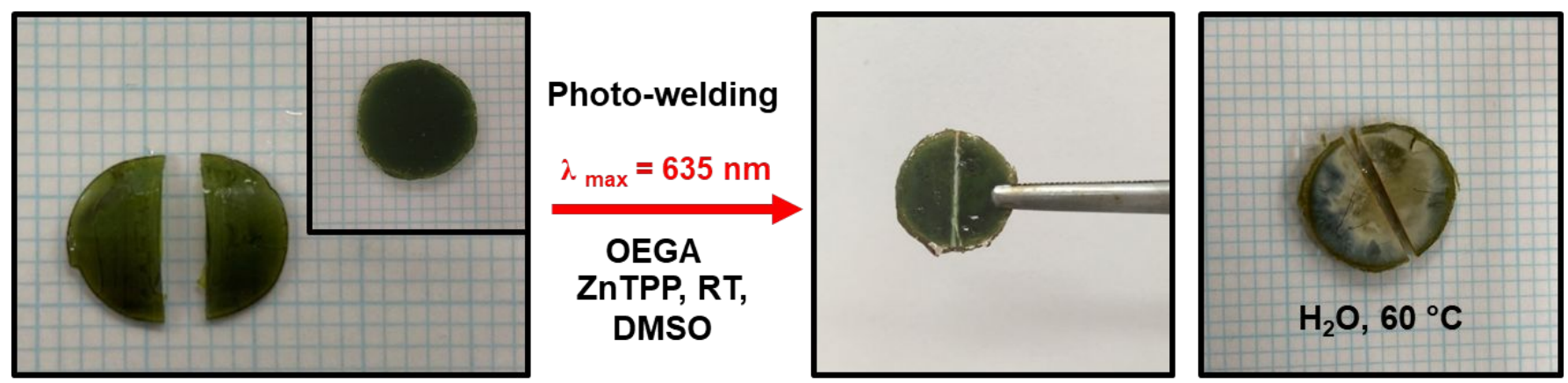

3D-PN-F-PNIPAAm

Figure S11. The optical images of the 3D-PN-F-PNIPAAm before and after a photo-welding process via a photoredox catalyzed polymerization at room temperature under red LED light in DMSO. 